\title{
Mumford-Shah Regularizer with Spatial Coherence
}

\author{
Erkut Erdem ${ }^{1}$, Aysun Sancar-Yilmaz ${ }^{2}$, and Sibel Tari ${ }^{1}$ \\ 1 Middle East Technical University, Department of Computer Engineering, \\ Ankara, TR-06531 \\ erkut@ceng.metu.edu.tr, stari@metu.edu.tr \\ 2 Aselsan Inc., Microwave and System Technologies Division, Ankara, TR-06172 \\ asancar@aselsan.com.tr
}

\begin{abstract}
As recently discussed by Bar, Kiryati, and Sochen in [3], the Ambrosio-Tortorelli approximation of the Mumford-Shah functional defines an extended line process regularization where the regularizer has an additional constraint introduced by the term $\rho|\nabla v|^{2}$. This term mildly forces some spatial organization by demanding that the edges are smooth. However, it does not force spatial coherence such as edge direction compatibility or edge connectivity, as in the traditional edge detectors such as Canny. Using the connection between regularization and diffusion filters, we incorporate further spatial structure into the regularization process of the Mumford-Shah model. The new model combines smoothing, edge detection and edge linking steps of the traditional approach to boundary detection. Importance of spatial coherence is best observed if the image noise is salt and pepper like. Proposed approach is able to deal with difficult noise cases without using non-smooth cost functions such as $L^{1}$ in the data fidelity or regularizer.
\end{abstract}

\section{Introduction}

Mumford and Shah [13] formulated image segmentation process as a functional minimization via which a piecewise smooth approximation of a given image and an edge set are to be recovered simultaneously. The Mumford-Shah energy is:

$$
E_{M S}(u, \Gamma)=\beta \int_{R}(u-g)^{2} d x+\alpha \int_{R \backslash \Gamma}|\nabla u|^{2} d x+\operatorname{length}(\Gamma)
$$

where

- $R \subset \Re^{2}$ is connected, bounded, open subset representing the image domain,

$-g$ is an image defined on $R$,

$-\Gamma \subset R$ is the edge set segmenting $R$,

$-u$ is the piecewise smooth approximation of $g$,

$-\alpha, \beta$ are the scale space parameters of the model. 
The first term in $E_{M S}(u, \Gamma)$ is the data fidelity term, which forces the solution $u$ to be as close as to the original image $g$. The other two terms are the regularization terms, which give preference to piecewise smooth images with simple edge sets. The unknown edge set $\Gamma$ makes the minimization mathematically difficult. A convenient approximation is suggested by Ambrosio and Tortorelli [2] following the $\Gamma$ convergence framework [7]. The basic idea is to introduce a smooth edge indicator function $v$ which is more convenient than the original edge indicator represented by the characteristic function $1-\chi_{\Gamma}$. The function $v$ depends on a parameter $\rho$, and as $\rho \rightarrow 0, v \rightarrow 1-\chi_{\Gamma}$. That is, $v(x) \approx 0$ if $x \in \Gamma$ and $v(x) \approx 1$ otherwise. Moreover, the cardinality of the edge set $\Gamma$ can be approximated by $\frac{1}{2}\left(\rho|\nabla v|^{2}+\frac{(1-v)^{2}}{\rho}\right)$. The new functional is as follows:

$$
E_{A T}(u, v)=\int_{R} \beta(u-g)^{2}+\alpha\left(v^{2}|\nabla u|^{2}\right)+\frac{1}{2}\left(\rho|\nabla v|^{2}+\frac{(1-v)^{2}}{\rho}\right) d x
$$

Notice that as $v \rightarrow 0$, the smoothness constraint in the piecewise smooth model is switched off. It is possible to interpret $v^{2}$ as an analog form of the line process introduced by Geman and Geman [12]. As shown by Bar et al. [3] and Teboul et al. [18], the Ambrosio-Tortorelli approximation of the Mumford Shah functional defines an extended line process regularization where the regularizer has an additional constraint introduced by the term $\rho|\nabla v|^{2}$. This term mildly forces some spatial organization by demanding that the edges are smooth. However, it does not force spatial coherence such as edge direction compatibility or edge connectivity. On the other hand, in the traditional approach, segmentation is defined as a sequential bottom-up process composed of the following three steps:

$$
\begin{aligned}
& \text { - smoothing, } \\
& \text { - edge detection, } \\
& \text { - edge linking. }
\end{aligned}
$$

The purpose of the last step is to force global consistency to locally detected edges in order to come up with a coherent edge set. Interestingly, this last step is what Mumford-Shah model or its Ambrosio-Tortorelli approximation lacks. The importance of spatial coherence can be best observed when the image contains impulse noise (Fig 1). Some works use Mumford-Shah regularizer or its modification $[17,1]$ for restoration in the presence of impulse noise. In [3, 4], Bar et al. present a very promising approach. However, the success of their method stems mostly from the use of robust data fidelity by replacing $L^{2}$ norm with $L^{1}$. Similarly, in [17], Shah uses $L^{1}$ norm for both the data fidelity and the regularizer. In fact, the use of non-smooth cost functions such as $L^{1}$ for the data fidelity term in order to deal with outliers and impulse noise is well motivated both theoretically and experimentally $[8,14,17]$. Teboul et al. [18] present a modification to (2), by replacing the quadratic cost $|\nabla v|^{2}$ with $L^{1}$ cost, which leads to singular diffusivity. Numerical difficulties are the cons of singular diffusivities [9]. The cost function choice in [18] also leads to directional smoothing. As explored by Weickert [19], directional smoothing can offer significant feature preserving 


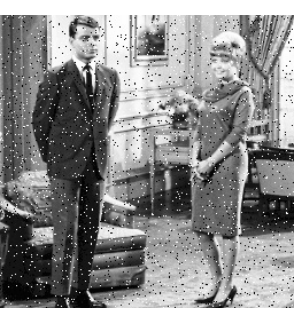

(a)

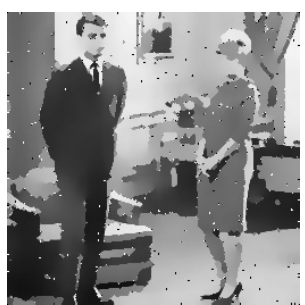

(b)

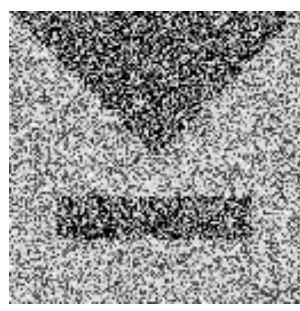

(c)

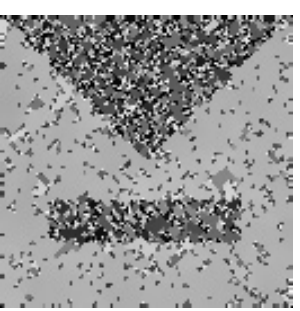

(d)

Fig. 1. Denoising cases which can not be handled by the Ambrosio-Tortorelli. (a)-(b) couple image corrupted with 5\% salt and pepper noise and its reconstruction using the Ambrosio-Tortorelli. (c)-(d) A noisy test image $-70 \%$ of the pixels are degraded with uniform noise- and its reconstruction using the AmbrosioTortorelli.

capabilities. However, the models get complicated and numerics is not as simple as in the case of isotropic diffusion.

In this work, we propose a modification to the Ambrosio-Tortorelli approximation of the Mumford-Shah functional, turning it into an edge-preserving regularization with spatial coherence. Key to our approach is the link between edge preserving regularization and diffusion filters $[15,16,6]$. Proposed model is a set of coupled linear diffusion equations. Hence, it is easy to implement.

We experimentally demonstrate denoising and edge preserving abilities of the proposed method. It can handle impulse noise and fill boundary gaps. Moreover, it can produce sharper results. Smoothed images obtained by the proposed method are qualitatively comparable to that are obtained by singular diffusion equations [9].

In the next section, we review the Ambrosio Tortorelli approximation and analyze it's behavior relevant to our developments given in $\S 3$. We present and discuss experimental results in $\S 4$. Finally, $\S 5$ is the summary and the conclusion.

\section{Gradient Descent Equations for Ambrosio-Tortorelli Energy}

Gradient descent equations for the Ambrosio-Tortorelli functional yield the following coupled PDEs:

$$
\begin{gathered}
\frac{\partial u}{\partial t}=\nabla \cdot\left(v^{2} \nabla u\right)-\frac{\beta}{\alpha}(u-g) ;\left.\quad \frac{\partial u}{\partial n}\right|_{\partial R}=0 \\
\frac{\partial v}{\partial t}=\nabla^{2} v-\frac{2 \alpha|\nabla u|^{2} v}{\rho}-\frac{(v-1)}{\rho^{2}} ;\left.\quad \frac{\partial v}{\partial n}\right|_{\partial R}=0
\end{gathered}
$$


where $\partial R$ denotes the boundary of $R$ and $n$ denotes the direction normal to $\partial R$. By alternating between these two biased diffusion equations, smooth image $u$ and the edge indicator function $v$ are simultaneously computed. Keeping $v$ fixed, (3) minimizes a convex quadratic functional given by

$$
\int_{R} \alpha v^{2}|\nabla u|^{2}+\beta(u-g)^{2}
$$

While the bias term in (3) or equivalently in (5) forces $u$ to be as close as to the original image $g$, the first term acts as an edge preserving regularizer. It smoothes the image with a smoothing radius proportional to the value of the function $v$ and $\frac{\alpha}{\beta}$. If there is an edge $(v \approx 0)$, no smoothing (diffusion) is applied.

On the other hand, keeping $u$ fixed, (4) minimizes a convex quadratic functional given by

$$
\int_{R} \rho|\nabla v|^{2}+\frac{1+2 \alpha \rho|\nabla u|^{2}}{\rho}\left(\frac{1}{1+2 \alpha \rho|\nabla u|^{2}}-v\right)^{2}
$$

The reciprocal relationship between $v$ and $|\nabla u|^{2}$ can be best observed in (6). Clearly, it asserts that $v$ function is nothing but a smoothing of

$$
\frac{1}{1+2 \alpha \rho|\nabla u|^{2}}
$$

with a blurring radius proportional to $\rho$ and reciprocal to $|\nabla u|$. Ignoring the smoothing, by letting $\rho \rightarrow 0[17,11]$,

$$
v \approx \frac{1}{1+2 \alpha \rho|\nabla u|^{2}}
$$

\section{Regularizer with Spatial Coherence}

The regularization of $v$ via $\nabla^{2} v$ term in (4) or equivalently $|\nabla v|^{2}$ term in (6) imposes some mild spatial organization $[3,18]$, by forcing $v$ to be smooth. However, this regularization does not force edge direction compatibility or edge continuity. Key contribution of our work is to modify the PDE given in (3) in a way that these constraints are incorporated. Our essential idea is to introduce a spatially varying function $c$, which increases for unpreferred spatial organization of the edges and decreases for the preferred ones. The value of the function appears as a multiplier in the diffusivity function giving (9).

$$
\frac{\partial u}{\partial t}=\nabla \cdot\left((c . v)^{2} \nabla u\right)-\frac{\beta}{\alpha}(u-g) ;\left.\quad \frac{\partial u}{\partial n}\right|_{\partial R}=0
$$

Function $(c v)^{2}$ can be seen as a modified edge indicator, which we compute directly without explicitly computing the scalar $c$. Regularization of $u$ is influenced by the modified indicator whereas the edge indicator $v$ itself remains the same. 
In the following subsections, we present two possible choices for the scalar function $c$, considering the edge coherency by means of directional consistency and edge continuity, respectively. Since the edge coherency corresponds a multiplier for the diffusivity function, it is possible to combine these two proposed functions into a single framework by taking the function $c$ as the product. The resulting framework considers the coherency of the edges by means of both the directional consistency and the edge continuity.

\subsection{Directional Consistency}

In the edge linking step of traditional boundary detection, edge pixels detected based on the magnitude of gradient are linked to give a connected edge set if their gradient directions are in agreement. Unlinked edge pixels are discarded. We induce such an effect in our diffusion model by increasing the relative persistence of the edge pixels, which are consistent with their neighbors, by increasing the diffusivity at inconsistent ones. We consider a coherency function $\phi(u)$ such that $\phi(u) \rightarrow 1$ on the preferred configurations and $\phi(u) \rightarrow 0$ on the incoherent configurations, and let $c$ has the following form:

$$
c=1+(1-\phi(u)) \frac{1-v}{v}
$$

First, notice that $c$ increases in proportion to the image gradient $|\nabla u|$, which is proportional to $\frac{1-v}{v}$ (See (8)). Second, notice that the overall diffusivity coefficient $(c . v)^{2}$ can be estimated as follows, without explicitly computing the variable $c$ :

$$
(c v)^{2}=(\phi(u) v+(1-\phi(u)) 1)^{2}
$$

The new diffusion coefficient is the square of the convex combination of $v$ and 1. Value of the diffusivity is bounded by 1 , attaining maximum value as $\phi(u) \rightarrow 0$, and decaying as $\phi(u) \rightarrow 1$ to a value determined by the edge indicator function $v$. We consider the following $\phi$ function, which simply measures the coherency as a function of edge directions.

$$
\phi\left(u_{i}\right)=\exp \left[\varepsilon\left(\frac{1}{\left|\eta_{s}\right|} \sum_{j \in \eta_{s}} \nabla u_{i} \cdot \nabla u_{j}-1\right)\right]
$$

where $\eta_{s}$ represents the neighborhood of pixel $i$ having $s$ neighbors. We define $\eta_{s}$ as $\pm s$ pixels along the orthogonal edge direction $\nabla u_{i}{ }^{\perp}$. The parameter $\varepsilon$ is a scalar, which determines the decay rate of the $\phi$ function. If the neighboring pixels are coherent (having similar edge directions), then the average angle between $\nabla u_{i}$ and $\nabla u_{j}$ 's is close to 0 making $\phi \rightarrow 1$.

\subsection{Edge Continuity}

The principle of edge continuity is used to eliminate streaking or breaking up of an edge contour due to noise or changing contrast. It is commonly referred as 
hysteresis due to successful application of threshold retardation in Canny edge detector [10]. In our diffusion model, we lower the diffusivity at pixels that correspond to broken parts of boundary segments to favor edge formation. There may be various choices for the selection of $c$. The important point is to decrease the modified diffusivity $(c v)^{2}$ if the neighboring site supports formation of an edge i.e having a low $v$ value. Recall (8) that gives the reciprocal relationship between $v$ and $|\nabla u|$. Decreasing diffusivity can be achieved by increasing the estimate of the image gradient, which is used in estimating the diffusivity. Therefore, a natural choice is to add an offset $h \in[0,1]$ indicating a support in favor of edge formation to the gradient term in the diffusivity estimate:

$$
(c v)^{2}=\left(\frac{1}{1+h+2 \alpha \rho|\nabla u|^{2}}\right)^{2}
$$

Such a choice yields

$$
c=\frac{1}{1+h v}
$$

In the discrete implementation of (9), diffusivities are estimated at midgrid points. Hence, $h$ should be computed as a support from a suitably chosen neighbor. For example, modified diffusivity $(c v)_{i+0.5, j}^{2}$ at a mid point between $(i, j)$ and $(i+1, j)$ may receive support in the form of either $\left(1-v_{i+0.5, j-1}\right)$ or $\left(1-v_{i+0.5, j+1}\right)$. Notice that lower the value of edge indicator at a neighboring site, higher the support it provides.

Adding spatial organization to energies defining regularization with line process has been previously proposed by Black and Rangarajan [5]. They define a local interaction energy that favors formation of unbroken contours. In [6], Black et al. derives the necessary update equations. If we let $\frac{v_{k}^{2}+v_{l}^{2}}{2}$ define a line process between site $k$ and site $l$, then our development becomes equivalent to that of Black et al. Thus, solving new coupled equations are qualitatively equivalent to modifying the Mumford-Shah with an additive term favoring unbroken contours as in Black and Rangarajan [5].

\section{Experimental Results}

The importance of directional consistency is best observed if the image contains impulse noise. Processing of the noisy couple image, shown in Fig 1(a), using the Ambrosio-Tortorelli and the new method are illustrated in Fig 2. Fig 2(a) and (b) illustrate smoothing obtained using the Ambrosio-Tortorelli with 400 iterations with different smoothing radius, $\frac{\alpha}{\beta}$. The result in Fig 2(a) is obtained with $\alpha=1, \beta=0.01, \rho=0.01$. When we increase the smoothing radius by choosing $\beta=0.001$, diffusion is highly severe that we even lose the head of the lady (Fig 2(b)). Yet, the noise is still present. If we use a regularization term which forces spatial coherence of the edges by means of the directional consistency, as discussed in $\S 3.1$, the image is denoised without blurring (Fig 2(c) and (d)). The perceptual difference between Fig 2(c) and (d) is in the sharpness level. The result in Fig 2(c) is obtained with the segmentation parameters 


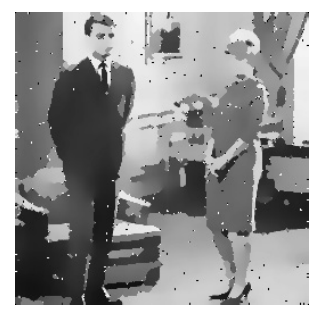

(a)

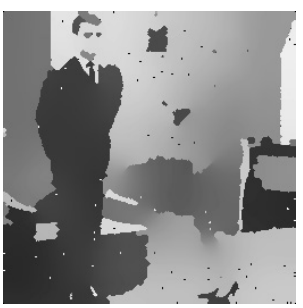

(b)

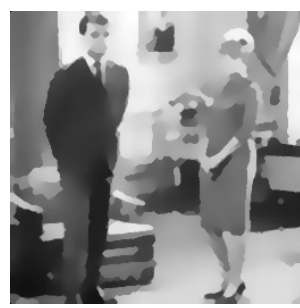

(c)

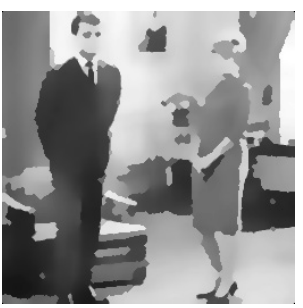

(d)

Fig. 2. Considering directional consistency eliminates impulse noise. (a)-(b) Reconstructions using the Ambrosio-Tortorelli with two different smoothing radius. Notice that the noise is still present even when we lose the head of the lady. (c)-(d) Reconstructions with directional consistency with two different sharpness levels. Notice that at comparable scales noise is completely eliminated.

specified for Fig 2(a) and the coherency parameters $s=2$ and $\varepsilon=0.25$ (this set of parameters are used for all of the experiments reported in the paper unless otherwise stated) with 50 iterations. For the result given in Fig 2(d), we use the same parameters except $\varepsilon=0.02$ and 300 iterations. The variable $\varepsilon$ determines the decay rate of the coherency function used in the segmentation process and therefore specifies the level of sharpness. For large $\varepsilon$ value, the decay rate is high and the edges are more smoothed out depending on the coherency. Hence, as observed, the resulting image is smoother. On the other hand, for small $\varepsilon$ values, we get sharper results.

Increasing the value of $\alpha$ while keeping $\frac{\alpha}{\beta}$ fixed means decreasing the penalty of the length term, yielding more detailed reconstruction. In Fig 3, the proposed modification is again tested with couple image with $5 \%$ salt and pepper noise (Fig 1(a)), however, forcing the reconstruction to be more detailed by the proper choice of parameters. Fig 3(a) is the outcome of the proposed modification after 20 iterations. On the other hand, Fig 3(c) is obtained by performing 50 iterations

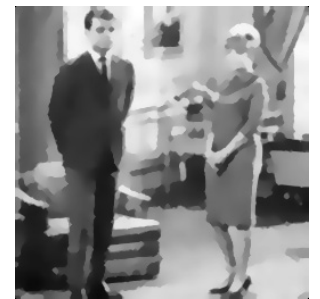

(a)

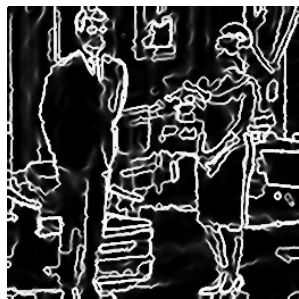

(b)

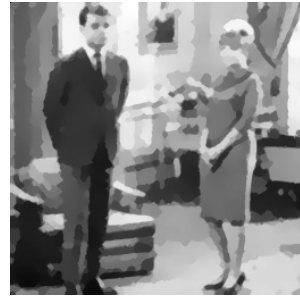

(c)

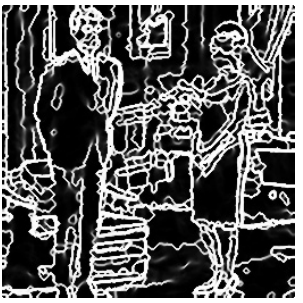

(d)

Fig. 3. $u$ and $1-v$ functions computed with $\alpha=1, \beta=0.01$ and $\alpha=4, \beta=0.04$ respectively. Even in more detailed reconstructions, modified scheme is able to remove noise completely 
with the same parameters except $\alpha=4, \beta=0.04$. The corresponding edge indicator functions are also shown in Fig 3(b) and (d) respectively. As they demonstrate, even the detailed reconstruction with $\alpha=4$ is noise free.

The example presented in Fig 4 illustrates the effect of edge continuity as described in $\S 3.2$. The results are obtained with 100 iterations. The reconstructions of the venice image shown in Fig 4(a) are presented in Fig 4 (b) and (c) together with the corresponding edge indicator functions. Fig 4(b) illustrates the outcome of the Ambrosio-Tortorelli whereas Fig 4(c) illustrates the result obtained by considering edge continuity. As it can be clearly seen from the zoomed indicator functions given in Fig 4(d), the modified scheme eliminates broken contours.
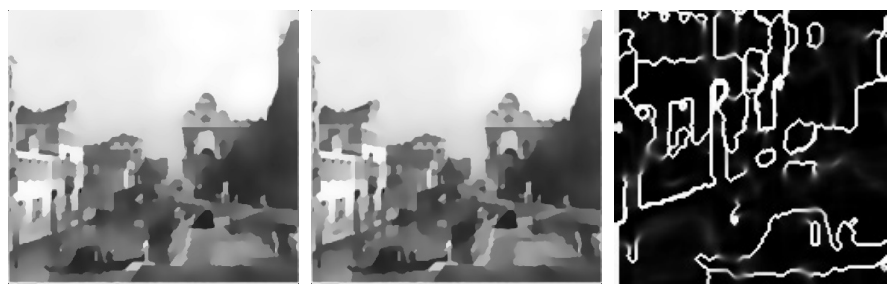

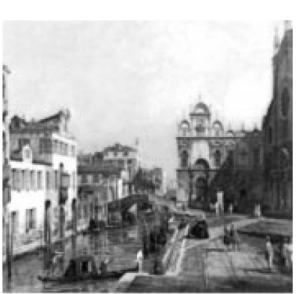

(a)

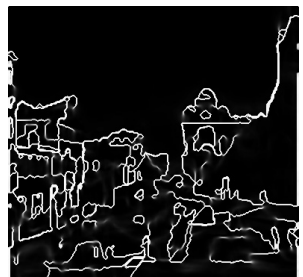

(b)

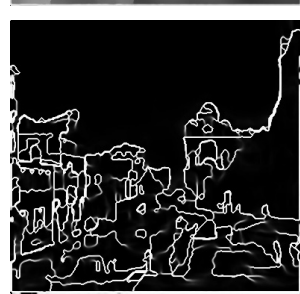

(c)

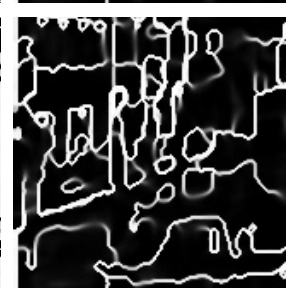

(d)

Fig. 4. Considering edge continuity eliminates broken contours. (a) input image. (b) Reconstruction using the Ambrosio-Tortorelli $(u$ and $1-v)$. (c) Reconstruction with modified scheme forcing edge continuity $(u$ and $1-v)(\mathrm{d})$ Details from the edge indicator functions given in (b) and (c) respectively.

In Fig 5, we demonstrate the results obtained with a regularization considering both the directional consistency and the edge continuity via the product of individual $c$ functions. The reconstruction results of venice image with $10 \%$ salt and pepper noise (Fig 5(a)) after 100 iterations are given in Fig 5(b)-(d). Fig 5(b) is the result obtained with edge continuity. As it can be clearly seen, the noise is not eliminated. Fig $5(\mathrm{c})$ is obtained with the modification which considers the directional consistency. Finally, Fig $5(\mathrm{~d})$ is the outcome of the combined framework which is not only noise free and but also having stronger edges.

In Fig 6, the combined framework is tested with a noisier image (Fig 1(c)). Fig 6(a) is the outcome of the Ambrosio-Tortorelli approximation after 500 iterations. Fig 6(b) is obtained with 150 iterations by using the modification, 


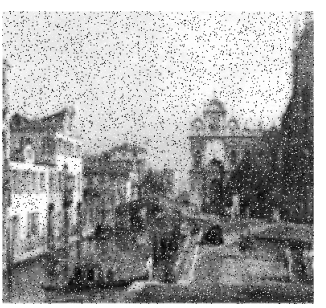

(a)

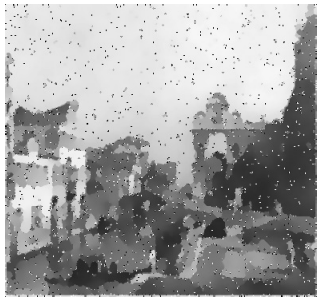

(b)

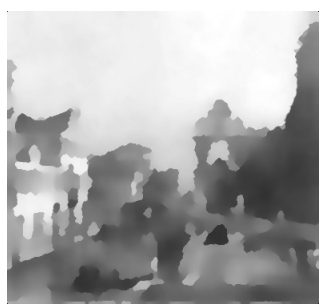

(c)

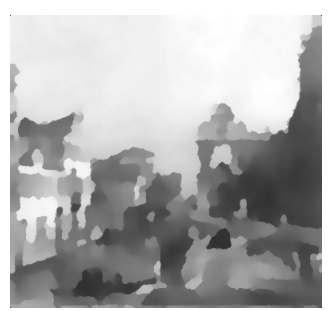

(d)

Fig. 5. Considering a combined framework eliminates both noise and the broken contours. (a) venice image corrupted with $10 \%$ salt and pepper noise. (b) Reconstruction with edge continuity. (c) Reconstruction with directional consistency. (d) Reconstruction using both edge continuity and directional consistency.

which considers only the directional consistency. Fig $6(\mathrm{c})$ is the outcome of the combined framework again after 150 iterations. Both reconstructions are qualitatively comparable to the ones obtained by means of singular diffusivities [9]. Notice that the two results are visually similar. This is due to the fact that the contrast is almost constant in the image. Hence, broken lines do not occur.

Mumford-Shah regularizer gives preference to piecewise smooth images with simple edge sets, without directly forcing edge direction compatibility or edge connectivity. Our final experiment demonstrates the potential of the modified model for textured images where the piecewise smooth assumption fails. As shown in Fig 7, directional consistency can also be used for further smoothing of the inhomogeneous textured regions that results in more coherent texture boundaries. Fig 7(a) is the input image sunflower. Fig 7(b) is the edge indicator function obtained by the Ambrosio-Tortorelli after 100 iterations with $\alpha=4$, $\beta=0.04$ and $\rho=0.001$. Even though the outer boundary separating leaves and seeds start to vanish, the inner boundary (small circles due to seeds) is clearly visible. Increasing $\alpha$ worsens the situations. On the other hand, when we consider

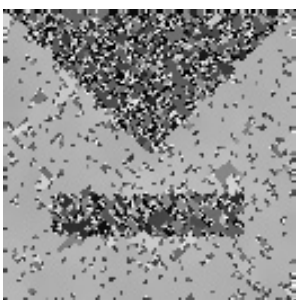

(a)

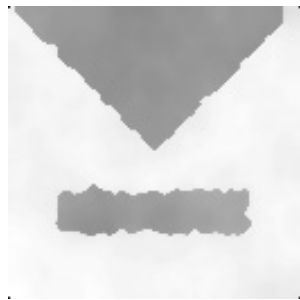

(b)

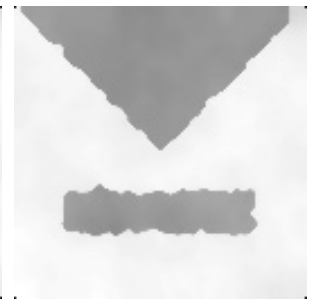

(c)

Fig. 6. A difficult denoising case. (a) Reconstruction using Ambrosio-Tortorelli. (b) Reconstruction with directional consistency. (c) Reconstruction using the combined framework. 


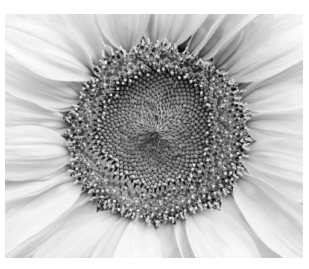

(a)

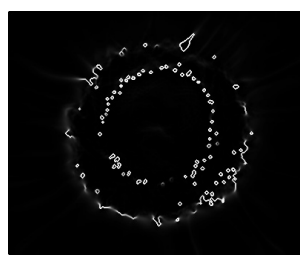

(b)

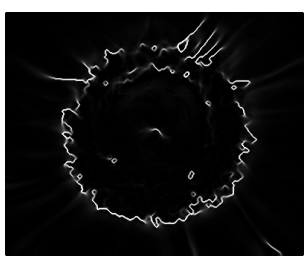

(c)

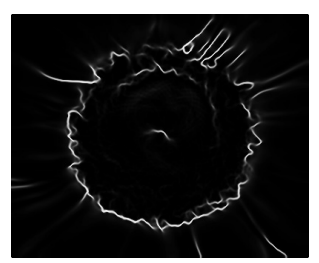

(d)

Fig. 7. An application to an image which violates the piecewise smooth assumption. (a) input image. (b) Edge indicator function computed using the AmbrosioTortorelli. Notice that when the outer boundary separating leaves and seeds are smoothed, the small circles due to texture gradient are still present. (c)-(d) Edge indicator functions computed with the directional consistency and the combined framework respectively. Observe that the edges due to texture gradient disappeared, yet the outer boundary is clearly visible.

the directional consistency of the edges with the parameters $\alpha=8, \beta=0.08$, $\rho=0.001, s=2$ and $\varepsilon=0.25$, the inner boundary is smoothed out and the outer boundary is present $(\mathrm{Fig} 7(\mathrm{c}))$. A reasonable result is also obtained by using the combined framework with the same parameters except $\varepsilon=2($ Fig $7(d))$.

\section{Summary and Conclusion}

Mumford-Shah model and its Ambrosio-Tortorelli approximation unify image smoothing and edge detection via coupling of two functions $u$ and $v$ representing smooth image and the edge indicator respectively. The edge indicator function $v$ defines an analog line process and its regularization imposes smoothness of the edge set. However, the model does not directly enforce spatial coherence as in the edge linking step of the traditional processing. We modify Ambrosio-Tortorelli model in its coupled diffusion equations form such that the regularization of $u$ is steered by the coherent edges. Our experiments demonstrate that the new regularization is able to remove difficult noise types and produce almost segmentation like results without using directional or singular diffusivities that arose from $L^{1}$ norm in the cost functions. In our experiments, we consider spatial coherency in terms of edge direction compatibility and edge continuity. However, further coherency criteria can be investigated, remaining in the same framework.

Acknowledgments This work is partially supported by the research grant TUBITAK-105E154 to S. Tari and TUBITAK-BAYG PhD scholarship to E. Erdem. 


\section{References}

1. R. Alicandro, A. Braides, and J. Shah. Free-discontinuity problems via functionals involving the $L^{1}$-norm of the gradient and their approximation. Interfaces and Free Boundaries, 1(1):17-37, 1999.

2. L. Ambrosio and V. Tortorelli. On the approximation of functionals depending on jumps by elliptic functionals via $\Gamma$-convergence. Commun. Pure Appl. Math., 43(8):999-1036, 1990.

3. L. Bar, N. Kiryati, and N. Sochen. Image deblurring in the presence of impulsive noise. Int. J. Comput. Vision, 70(3):279-298, 2006.

4. L. Bar, N. Sochen, and N. Kiryati. Image deblurring in the presence of salt-andpepper noise. In Scale-Space, pages 107-118, 2005.

5. M. J. Black and A. Rangarajan. On the unification of line processes, outlier rejection, and robust statistics with applications in early vision. Int. J. Comput. Vision, 19(1):57-91, 1996.

6. M. J. Black, G. Sapiro, D. H. Marimont, and D. Heeger. Robust anisotropic diffusion. IEEE Trans. Image Processing, 7(3):421-432, March 1998.

7. A. Braides. Approximation of Free-discontinuity Problems. Lecture Notes in Mathematics, Vol. 1694. Springer-Verlag, 1998.

8. T. Brox, A. Bruhn, N. Papenberg, and J. Weickert. High accuracy optical flow estimation based on a theory for warping. In $E C C V$ (4), pages 25-36, 2004.

9. B. Burgeth, J. Weickert, and S. Tari. Minimally stochastic schemes for singular diffusion equations. In X.-C. Tai, K.-A. Lie, T. F. Chan, and S. Osher and, editors, Image Processing Based on Partial Differential Equations, Mathematics and Visualization, pages 325-339. Springer Berlin Heidelberg, 2006.

10. J. Canny. A computational approach to edge detection. IEEE Trans. Pattern Anal. Mach. Intell., 8(6):679-698, 1986.

11. T. Chan and L. Vese. Variational image restoration and segmentation models and approximations. UCLA, CAM-report 97-47, September, 1997.

12. S. Geman and D. Geman. Stochastic relaxation, gibbs distributions, and the bayesian restoration of images. IEEE Trans. Pattern Anal. Mach. Intell., 6:721639, 1984.

13. D. Mumford and J. Shah. Optimal approximations by piecewise smooth functions and associated variational problems. Commun. Pure Appl. Math., 42(5):577-685, 1989.

14. M. Nikolova. A variational approach to remove outliers and impulse noise. $J$. Math. Imaging Vis., 20(1-2):99-120, 2004.

15. O. Scherzer and J. Weickert. Relations between regularization and diffusion filtering. J. Math. Imaging Vis., 12(1):43-63, 2000.

16. J. Shah. Segmentation by nonlinear diffusion. In CVPR, pages 202-207, 1991.

17. J. Shah. A common framework for curve evolution, segmentation and anisotropic diffusion. In CVPR, pages 136-142, 1996.

18. S. Teboul, L. Blanc-Fraud, G. Aubert, and M. Barlaud. Variational approach for edge preserving regularization using coupled pde's. IEEE Trans. Image Processing, 7(3):387-397, March 1998.

19. J. Weickert. Coherence-enhancing diffusion filtering. Int. J. Comput. Vision, 31(23):111-127, 1999. 